\title{
Rescue Argon Plasma Coagulation-Cutting for Removal of Stuck Biliary Fully Covered Self-Expanding Metal Stent
}

\author{
Vincent Zimmer ${ }^{1,2}$ \\ 1) Department of Medicine, Marienhausklinik St. Josef Kohlhof, Neunkirchen, 2) Department of Medicine II, Saarland University Medical \\ Center, Saarland University, Homburg, Germany
}
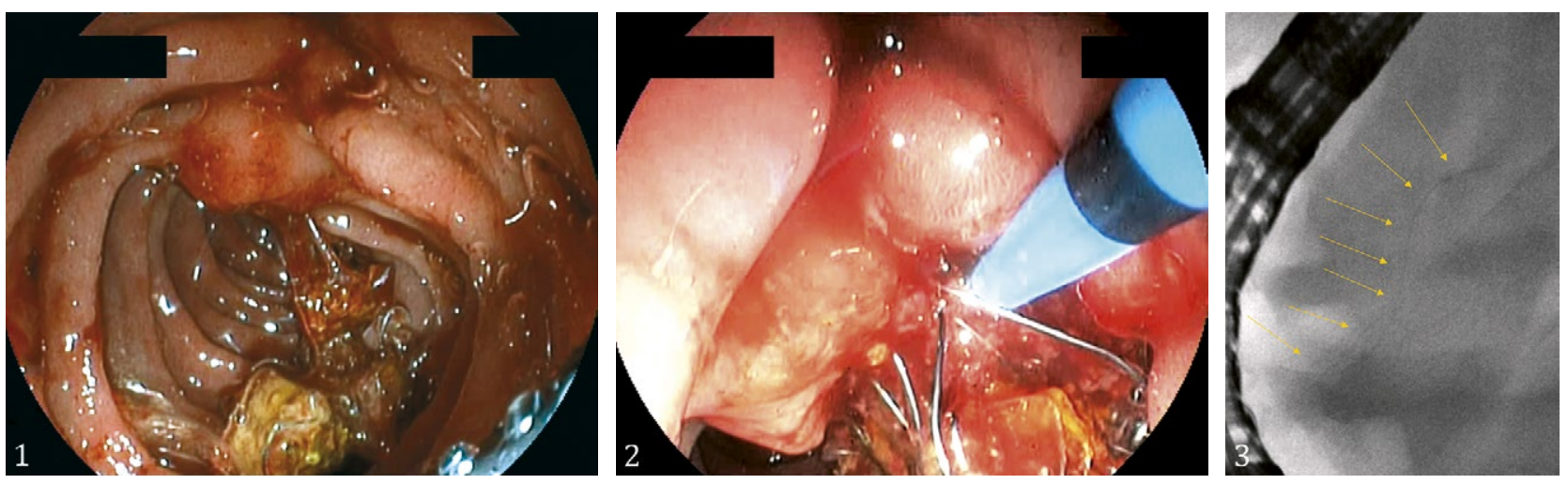

Biliary fully covered self-expanding metal stents (fcSEMS) have been widely embraced for endoscopic treatment over a broad spectrum of biliary strictures, including those related to chronic pancreatitis [1]. While available human and animal data indicate minimal stent-related tissue overgrowth, endoscopic removability rates from larger-scale studies and real world-experience are high [2]. Notwithstanding, a small fraction of stents may cause difficulty during extraction, which is particularly challenging in cases of mechanical stent deformation and wedging. Argon-plasma coagulation (APC) cutting has mostly been reported to luminally trim excessively long stents; however, to the best of my knowledge, this has not been described as a rescue technique in the specific clinical situation of a tricky fcSEMS extraction.

A 50-year old male patient with chronic pancreatitis, underwent biliary fcSEMS placement $(60 \times 10-\mathrm{mm}$, Taewong Medical) eight months before. During the scheduled removal the stent progressively unwind due to an initially unappreciated focal proximal overgrowth, resulting in marked distortion and stucking (Fig. 1A). Endoscopic cutting of stuck stent meshes was performed cautiously by argon plasma coagulation (100 $\mathrm{W}$, gas flow $1.5 \mathrm{~L} / \mathrm{min}$ ) and the "stent package" orally extracted (Fig. 2). One cut stent strut was left in place as indicated by fluoroscopy (Fig. 3) and due to papillary edema and minor hemobilia, a temporary plastic stent was placed to secure biliary drainage. Four weeks later the metal thread was dislodged from the bile duct, using an 8-wire Dormia basket, grasped in the duodenal lumen and orally extracted.
While removability of fcSEMS is typically uncomplicated and straightforward, in a minor fraction of patients unravelling may occur, causing difficulty in extraction. High-grade distortion and unraveling of fcSEMS has been reported to occur in about $2 \%$ of patients in a multicenter trial [3]. This was considered to be most likely due to an initially unappreciated focal proximal overgrowth of one or two stent struts. To this end, APC cutting was performed resulting in successful removal.

Corresponding author: Vincent Zimmer, vincent.zimmer@gmx.de

Conflicts of interest: None to declare.

\section{REFERENCES}

1. Lakhtakia S, Reddy N, Dolak W, et al. Long-term outcomes after temporary placement of a self-expanding fully covered metal stent for benign biliary strictures secondary to chronic pancreatitis. Gastrointest Endosc 2020;91:361-369.e3. doi:10.1016/j.gie.2019.08.037

2. Deviere J, Nageshwar Reddy D, Puspok A, et al. Successful management of benign biliary strictures with fully covered self-expanding metal stents. Gastroenterology 2014;147:385-395. doi:10.1053/j. gastro.2014.04.043

3. Kahaleh M, Brijbassie A, Sethi A, et al. Multicenter trial evaluating the use of covered self-expanding metal stents in benign biliary strictures: time to revisit our therapeutic options? J Clin Gastroenterol 2013;47:695-699. doi:10.1097/MCG.0b013e31827fd311 